\title{
Determinants of harmful use of alcohol among urban slum dwelling adults in Kenya
}

\author{
Mariam Gitatui ${ }^{1}$, Samuel Kimani ${ }^{1}$, Samuel Muniu ${ }^{2}$, Okubatsion Okube ${ }^{3}$
}

1. University of Nairobi, School of Nursing Sciences.

2. Aidspan, Policy Analysis.

3. Catholic University of Eastern Africa, Department of Nursing.

\begin{abstract}
Background: Harmful alcohol use is a public health problem associated with negative health and socio-economic impacts. However, patterns and dynamics of alcohol use among slum-dwellers in Kenya are poorly understood.

Objective: To establish determinants of harmful alcohol use among adults in an urban slum setting in Kenya.

Materials and methods: Cross-sectional study involving a consecutively selected sample (N=215) from Githurai, in Nairobi. A pre-tested questionnaire that captured data on socio-demographics, drinking patterns, type, reasons, initiator, and support system.
\end{abstract}

Results: Of the respondents, those above 31 years, married, separated/divorced/widowed, of high education, earning above 50 USD, and from dysfunctional families consumed more alcohol. Low earners consumed $(p<0.05)$ unrecorded while high earners drank $(p<0.001)$ recorded alcohol. Adults from families with a drinking father and sibling consumed more alcohol $(p=0.001)$. Single, low educational attainment/earners, and those in dysfunctional families $(p<0.05)$ drank due to stress and reported alcohol-related problems. Young, unmarried, and casual laborers were introduced $(\mathrm{p}<0.05)$ to alcohol by friends.

Conclusion: Socio-demographic, economic, familial, social interactions, and stress are associated with harmful alcohol use among adults from slums calling for interventions targeting these factors.

Keywords: Determinants; urban slum dwelling; alcohol use; alcohol abuse; adults; informal.

DOI: https://dx.doi.org/10.4314/ahs.v19i4.12

Cite as: Gitatui M, Kimani S, Muniu S, Okube O. Determinants of harmful use of alcohol among urban slum dwelling adults in Kenya. Afri Health Sci. 2019;19(4):2906-2925. https:// dx.doi.org/10.4314/abs.v19i4.12

\section{Background}

Alcohol use and/or abuse has been in existence since antiquity. Harmful use of alcohol is associated with social, economic, psychological and physical consequences on individual, family, and the community ${ }^{1,2}$, with increased propensity for toxicity, injuries, and violence ${ }^{3,4}$. Approximately 2.5 million deaths occur annually attributed to alcohol consumption resulting to significant morbidity, disability, violence, child neglect, abuse, and economic deprivation ${ }^{4}$. Indeed, harmful use of alcohol ranks top five risk factors for chronic diseases, disability and death globally ${ }^{4,5}$. Specifically, alcohol use is linked to heart dis-

\section{Corresponding author: \\ Samuel Kimani, \\ University of Nairobi, \\ School of Nursing Sciences, \\ Email: tkimani@uonbi.ac.ke}

eases, liver cirrhosis, cancers, high blood pressure, high cholesterol and their attendant morbidity and mortality ${ }^{6}$, whose direct relationship has been clearly established. Furthermore, there is increased risk of accidental injuries, suicides, murders, and domestic violence associated with alcohol use ${ }^{7}$. Moreover, studies have demonstrated that children of alcoholics have increased risk for violent behavior, ${ }^{9}$ perform poorly in academics ${ }^{10}$ and are vulnerable to higher incidence of depression, anxiety, stress and lower self-esteem among some of the serious long term sequelae ${ }^{11,9}$.

Alcoholic beverages consumed across regions are either recorded (wine, beer and spirits) or unrecorded (allegedly cheaper homemade undocumented brands) depending on culture and settings ${ }^{4}$. The magnitude of alcohol consumption varies geographically, with highest quantities of recorded alcohol use reported in high-income countries, while unrecorded type is consumed in low income nations ${ }^{4}$. Regionally, Southern African countries (Namibia

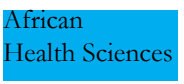

C) 2019 Gitatui et al. Licensee African Health Sciences. This is an Open Access article distributed under the terms of the Creative commons Attribution License (https://creativecommons.org/licenses/BY/4.0), which permits unrestricted use, distribution, and reproduction in any medium, provided the original work is properly cited.

African Health Sciences Vol 19 Issue 4, December, 2019 
and republic of South Africa) top in alcohol consumption compared to their neighbouring nations ${ }^{4}$. In East Africa, Kenya (31.7\%) and Uganda (28.6\%) have highest alcohol consumption rates, respectively ${ }^{4,8,9}$, with proportions higher in urban relative to rural settings ${ }^{9,10}$.

The Kenyan urban, slum, and informal settings dwellers consume more unrecorded alcohol, sometimes risking their lives ${ }^{9}$. Substantial consumption of unrecorded alcohol is attributed to high prices, taxes, and legal requirements associated with recorded alcoholic drinks as well as it is a cultural norm ${ }^{4,9}$. The consumption of the unrecorded alcohol is associated with social-cultural activities and less stringent measures which sometimes may promote excessive intake. Unrecorded alcohol is implicated in increased risk of harm because of unknown and potentially dangerous impurities or contaminants ${ }^{4,11-13}$. The alcohol-related complications are related to the type of alcohol (recorded vs unrecorded), amount, frequency and the health status of the consumer. The complications manifest even after several hours after taking alcohol and are reported as headache, fatigue and irritability as well as the need to drink in the morning for one to start working- dependence syndrome ${ }^{14}$. It is worth noting however, that consumption and the attendant alcohol-related complications are disproportionally higher in men compared to women, with $6.2 \%$ all male vs $1.1 \%$ female deaths attributed to alcohol ${ }^{4}$. The gender differences are attributed to cultural acceptability and economic capability in favour of men thus high consumption among men ?

Locally, the prevalence of alcohol-related problems is high with an estimated 5.8\% of adult Kenyan males (1564 years old) having some level of alcohol dependency and $2.4 \%$ categorized as abusing alcohol ${ }^{4,10,15,16}$. Alcohol-related problems are attributed to unrecorded alcohol, an issue that has a national dimension with urban slum settlements significantly affected because of affordability 9, 11, 15. The unrecorded alcoholic beverages that are commonly consumed in these settings are chang'aa and busaa. Chang'aa is a high (15.3-34\%) alcohol content spirit-like clear drink made by fermenting a mixture of corn/sorghum/millet and sugar for a week, followed by distillation. The busaa on the other hand, is a malt liquor with an alcohol content of $3.9 \%-5.4 \%$ made from fermenting corn flour/sorghum/millet over a shorter period of about two days ${ }^{9,17,18}$. These alcoholic beverages are prone to abuse because they are affordable, available and culturally acceptable products that are consumed during community activities and special occasions 4, 9, 10, 15, 16,19.

Alcohol use and/or abuse commences during adolescence and young adulthood progressing into adulthood 1 . This age cohort has been reported to engage in hazardous and harmful alcohol use practices characterized by regular, intoxication, and binge drinking ${ }^{20-22}$. Their alcohol use is influenced by family and social environment they live and grow ${ }^{23}$. They commence alcohol consumption within the family, with parental knowledge ${ }^{24}$. Thus, there are family related factors that promote alcohol use for example; family conflict, poor communication, parental drinking, and permissiveness ${ }^{25}$. Indeed, evidence show a parent who abuses alcohol is a risk factor for children becoming alcoholics ${ }^{26}$, while having siblings who misuse alcohol is even stronger risk factor towards other sibling drinking ${ }^{27-29}$. Additionally, family conflict have been linked to adolescent alcohol abuse either directly ${ }^{30}$, or through reduced effectiveness of parental monitoring ${ }^{31}$. Importantly, the parental attitude that favors antisocial behavior tends to increase the risk of children abusing alcohol ${ }^{32}$, 33. Similarly, peer effect, wider social, environmental and legislative contexts influence the use of alcohol ${ }^{34}$. The peers and social norms determine adolescent alcohol use behaviour ${ }^{35-38}$. In this regard, as one grows social contacts expands and friends' approval influence consumption of alcohol ${ }^{39}$. However, parental influence has been shown to remain stronger particularly where family relationships are perceived to be close by the child ${ }^{40,41}$.

Recently, changes in trends and patterns associated with alcohol use such as; drinking at young age, increased amount consumed, and lack of support system to address impacts of alcohol use have been observed ${ }^{42}$. Taken together, the aforementioned depicts harmful alcohol use is a socio-economic and public health problem affecting the young productive population. Indeed, evidence link young age drinking to alcohol dependency and related social economic impacts. Therefore, delayed initiation to alcohol significantly impede alcohol misuse over a longer term ${ }^{43}$. Importantly, alcohol-related health consequences are inversely related to the age when drinking commenced ${ }^{44,45}$, availability of support systems as well as directly linked to the amount ${ }^{46}$. The factors surrounding pervasive alcohol use and/or abuse are poorly understood in Kenya despite alcoholism having reached alarming levels. High alcohol abuse has been thought to 
contribute to increased morbidity and mortality among Kenyan adult men 10,15,16. Studies delineating the factors influencing alcohol use patterns in informal (slums) settlements in Kenya are lacking. Thus, this study sought to establish the determinants of alcohol consumption among young adults in an urban slum setting in Kenya.

\section{Materials and methods Study setting}

The study was conducted in Soweto slum, an informal settlement located in Githurai sub-location, East of Nairobi City and County. The slum is located approximately 12 KM from Nairobi central business district, 300 meters off Thika super highway. The slum sits on approximately 13 acres of land, borders Thika super highway to the South, Maziwa estate to the North, Githurai 44 to the East, Farmers choice industries and Kahawa Army barracks to the West. The area has 3 registered bars and 10 chang'aa brewing homesteads doubling up as drinking places. The slum is inhabited by about 10, 000 people among them $40 \%$ are adults ${ }^{47}$.

\section{Study design and sampling}

This was a cross-sectional study conducted between April and July 2016 involving 215 adults (over 18 years) who reported to be regular alcohol drinkers regardless of the amount from Soweto Slum in Githurai Nairobi, Kenya. A two stage cluster sampling was used to select one Sub-location and five data collection sites that were proximal to the drinking places. Consecutive sampling that is considered the best type onon-probability sampling with good representation of entire population, was used to include all accessible alcohol users as part of the sample. This is a form of convenient sampling method where participants are selected in order of appearance. The sample size was calculated based on the recommendation by Mugenda and Mugenda ${ }^{48}$ which indicates that if the target population is less than 10,000 , the a sample size of 10 to $30 \%$ is adequate. For this study $20 \%$ was considered adequate and since the target population was 4,000 people, the minimum sample size required was 200 respondents. The participants were recruited from the five data collection sites resulting with 43 respondents from each site consecutively sampled until the total number was achieved.

\section{Study participants}

The participants for this study were alcohol users (self-re- ported) male and female aged 18 years and above. All men and women who reported to be current alcohol users of alcohol on at least one day per week and who availed themselves at the data collection sites were eligible for the study. The participants were approached for recruitment by research assistants who had been recruited for their role in the community as mobilizers, social workers or peer educators to participate in collecting data for this study. The research assistants were trained on the study methodology, consent and recruitment processes. The research assistant conducted face-to-face interviews using the language participant was most comfortable with. All alcohol users who met the inclusion criteria were informed and explained to about the objective, procedure of the study, and informed consent process. In this regard, the study participants provided their individual verbal and written informed consent before participating in the study. The content of the informed consent was read by or to each of respondent depending on one's choice or whether one could read or not. The informed consent process involved explanation by the researcher on: what the study was all about; aim of the study and methods for data collection; anticipated benefits including non-monetary compensation; potential risks in which case no risks were involved apart from being interviewed; measures to ensure confidentiality and anonymity of the information; voluntarism and the right to withdraw from the study it at any time without reprisal; institutional affiliations and contacts of the researchers, as well as contacts for ethical review committee person to report any adverse outcome/events. All those who agreed to participate in the study after the consent process were requested to signed a consent certificate and were recruited to participate in the study. Participation was limited to adults aged over 18 years. Those recruited received a soft drink for participating in the study.

\section{Data collection}

Data were collected using a researcher-assisted structured questionnaire. The questionnaire was structured into socio-demographic characteristics as well as closed ended questions to capture quantitative data on alcohol consumption in the past thirty days. Specifically, the components of the questionnaire included; socio-demographic characteristics, drinking patterns, type of drink, other drugs, reasons for drinking, persons that introduced respondent to alcohol, support system for alcohol 
problems, and reported feelings after waking up among others. The questionnaire was developed following a discussion with the study investigators on the variables that are pertinent in the issue of alcohol use. Once developed the question was shared with a panel of experts who had knowledge of the topic and the emerging issues were further refined and included in the questionnaire. Finally, the questionnaire was validated by a group of the investigators and experts ready for pre-testing. This process was to guarantee validity and reliability of the questionnaire. The questionnaires were pre-tested among 10 respondents who reported to have been users of alcohol sampled from the neighbouring Githurai area. The responses from pre testing were assessed and used to review the final data collection tool.

\section{Data analyses}

Data were organized, screened and checked for completeness. Thereafter coding, input into computer, and cross checking against the original data set for accuracy was conducted. Data were analyzed using computer software (SPSS Ver. 22) for which descriptive and inferential statistical outputs were generated and reported appropriately. Categorical data were summarized into proportions and presented in frequency tables. To determine relationships between various variables Chi-square test of independence and Fisher's Exact test were performed. For variables that were found to have a significant difference and had more than two categories a logistic regression waperformed to determine the group responsible for the difference. Numeric data was summarized into mean and standard deviations. The difference between variables was determined using independent t-test and one-Way ANOVA.

\section{Ethical consideration}

Ethical approval for the study was obtained from Kenyatta National Hospital-University of Nairobi Ethical Review Committee (KNH-UoN ERC) (Approval number UP365/05/2016). Permission to conduct the study in the slum area was sought and granted by the Githurai Assistant County Commissioner (Ref. KASD/AD$\mathrm{M} / 1 / 1 \mathrm{VOL} .5 / 192)$. Both verbal and written consents were obtained from respondents after comprehensive explanation.

\section{Results}

\section{Socio-demographic characteristics of the respon- dents}

Of the respondents, majority were males, aged 30 years or below (Table 1), married (42.3\%), single (42.3\%) or separated/divorced/widowed (15.3\%). Most of the respondents had attained primary education, were self-employed, and earned monthly income of less than USD 50 (Ksh: 5, 000), and described their families as happy. 
Table 1: Socio-demographic characteristics of the respondents

\begin{tabular}{|c|c|c|}
\hline Variable & Frequency & Percent \\
\hline \multicolumn{3}{|l|}{ Age } \\
\hline$\leq 30$ years & 111 & 51.6 \\
\hline $31-40$ years & 76 & 35.3 \\
\hline Over 40 years & 28 & 13.0 \\
\hline \multicolumn{3}{|l|}{ Gender } \\
\hline Female & 43 & 20.0 \\
\hline Male & 172 & 80.0 \\
\hline \multicolumn{3}{|l|}{ Marital status } \\
\hline Single & 91 & 42.3 \\
\hline Married & 91 & 42.3 \\
\hline Separated/Divorced/Widowed & 33 & 15.3 \\
\hline \multicolumn{3}{|l|}{ Education } \\
\hline None - Primary & 142 & 66.0 \\
\hline Post-primary & 73 & 34.0 \\
\hline \multicolumn{3}{|l|}{ Employment status } \\
\hline Casual laborer & 31 & 14.6 \\
\hline Employed & 18 & 8.5 \\
\hline Self-employed & 164 & 77.0 \\
\hline \multicolumn{3}{|l|}{ Monthly earning (USD) } \\
\hline Less than 50 & 131 & 61.8 \\
\hline $50-100$ & 51 & 24.1 \\
\hline Over 100 & 30 & 14.2 \\
\hline \multicolumn{3}{|l|}{ Family description } \\
\hline Abusive/broken home & 34 & 16.0 \\
\hline Happy home & 178 & 84.0 \\
\hline
\end{tabular}

Relationship between socio-demographic characteristics and alcohol use

Analysis of the relationship between social demographic factors and the patterns of alcohol use revealed that respondents who consumed more than three drinks were more likely $(\mathrm{p}<0.05)$ to be older $(\mathrm{OR}=5.8,95 \% \mathrm{CI}$ : $2.3-14.2$ and OR $=2.6,95 \%$ CI: 1.1 - 6.4), married $(\mathrm{OR}$ $=8.3,95 \%$ CI: $3.3-21.1)$, separated $/$ divorced/widowed
$(\mathrm{OR}=2.8,95 \% \mathrm{CI}: 1.3-6.5)$, had attained post primary education $(\mathrm{OR}=2.1,05 \% \mathrm{CI}: 1.1-3.8)$, and of income above 50 USD (OR $=5.8,95 \%$ CI: $2.5-13.8$ and OR $=8.8,95 \%$ CI: $3.1-25.5$ ) (Table 2). Specifically, those aged $31-40$ years were 5.8 times likely to consume more than three drinks compared with other age groups. The married were 8.3 times likely to consume more than three drinks. Those with post-primary education were 2.1 times 
likely to consume more than three drinks. Respondents earning USD 50 above were likely to consume more than three drinks in one sitting. Although marginally significant, respondents from happy homes were less likely to consume three alcoholic drinks per day.

Table 2: Relationship between demographic characteristics and daily alcohol use

\begin{tabular}{|c|c|c|c|c|}
\hline \multirow[b]{2}{*}{ Characteristic } & \multicolumn{2}{|c|}{ Drinks per day } & \multirow[b]{2}{*}{ Total } & \multirow[b]{2}{*}{$\operatorname{AOR}(95 \% \mathrm{CI})$} \\
\hline & $\begin{array}{l}\text { More } \\
\text { than } 3 \\
\text { drinks }\end{array}$ & $\begin{array}{c}1-3 \\
\text { drinks }\end{array}$ & & \\
\hline \multicolumn{5}{|l|}{ Age } \\
\hline Less than 30 years & $18(16.7)$ & $90(83.3)$ & $108(100)$ & Reference \\
\hline $31-40$ years & $23(30.7)$ & $52(69.3)$ & $75(100)$ & $5.8(2.3-14.2)^{*}$ \\
\hline Over 40 years & $15(53.6)$ & $13(46.4)$ & $28(100)$ & $2.6(1.1-6.4)^{*}$ \\
\hline \multicolumn{5}{|l|}{ Gender } \\
\hline Female & $8(18.6)$ & $35(81.4)$ & $43(100)$ & Reference \\
\hline Male & $48(28.6)$ & $120(71.4)$ & $168(100)$ & $1.8(0.9-4.0)$ \\
\hline \multicolumn{5}{|l|}{ Marital status } \\
\hline Single & $11(12.6)$ & $76(87.4)$ & $87(100)$ & Reference \\
\hline Married & $27(29.7)$ & $64(70.3)$ & $91(100)$ & $8.3(3.3-21.1)^{*}$ \\
\hline Separated/Divorced/Widowed & $18(54.5)$ & $15(45.5)$ & $33(100)$ & $2.8(1.3-6.5)^{*}$ \\
\hline \multicolumn{5}{|l|}{ Education } \\
\hline None - Primary & $30(21.6)$ & $109(78.4)$ & $139(100)$ & Reference \\
\hline Post-primary & $26(36.1)$ & $46(63.9)$ & $72(100)$ & $2.1(1.1-3.8)^{*}$ \\
\hline \multicolumn{5}{|l|}{ Religion } \\
\hline Christians & $41(21.9)$ & $146(78.1)$ & $187(100)$ & Reference \\
\hline Islam & $12(70.6)$ & $5(29.4)$ & $17(100)$ & $2.7(0.6-12.4)$ \\
\hline Others & $3(42.9)$ & $4(57.1)$ & $7(100)$ & $0.3(0.1-1.9)$ \\
\hline \multicolumn{5}{|l|}{ Monthly earning (USD) } \\
\hline Less than 50 & $28(21.9)$ & $100(78.1)$ & $128(100)$ & Reference \\
\hline $50-100$ & $8(15.7)$ & $43(84.3)$ & $51(100)$ & $5.8(2.5-13.8)^{*}$ \\
\hline Over 100 & $18(62.1)$ & 11(37.9) & $29(100)$ & $8.8(3.1-25.5)^{*}$ \\
\hline \multicolumn{5}{|l|}{ Family description } \\
\hline Abusive/broken home & $13(39.4)$ & $20(60.6)$ & $33(100)$ & Reference \\
\hline Happy home & $41(23.4)$ & $134(76.6)$ & $175(100)$ & $0.5(0.2-1.0)^{\dagger}$ \\
\hline Total & $54(26)$ & $154(74)$ & $208(100)$ & \\
\hline
\end{tabular}

Relationship between socio-demographics factors and alcohol use per week

Respondents reported consuming alcohol on average 4.15 \pm 2.8 (Mean $\pm \mathrm{SD}$ ) days per week. A One-Way ANOVA revealed age, marital status, religion, employment status, and monthly earnings were associated with more days of alcohol consumption per week (Table 3). Respondents who were older $(\mathrm{F}(2,210)=5.786, \mathrm{p}=0.004)$, separated, widowed or divorced $(\mathrm{F}(2,210)=5.766, \mathrm{p}=0.004)$, employed $(\mathrm{F}(2,208)=6.016, \mathrm{p}=0.003)$, and higher earners $(\mathrm{F}(2,207)=3.505, \mathrm{p}=0.032)$ were more likely to consume alcohol in most days of the week. 
Table 3: Relationship between demographic characteristics and weekly alcohol use

\begin{tabular}{|c|c|c|c|c|c|c|}
\hline Variable & $\mathrm{N}$ & $\begin{array}{l}\text { Mean drinking } \\
\text { days per week }\end{array}$ & $\mathrm{SD}$ & $\mathrm{F}$ & $\mathrm{df}$ & $\mathrm{P}$ \\
\hline Age & & & & 5.786 & 2,210 & 0.004 \\
\hline Less than 30 years & 110 & 3.60 & 2.72 & & & \\
\hline $31-40$ years & 75 & 4.51 & 2.83 & & & \\
\hline Over 40 years & 28 & 5.39 & 2.44 & & & \\
\hline Total & 213 & $4.15(4.50)$ & 2.79 & & & \\
\hline Gender & & & & 0.919 & 1,211 & 0.339 \\
\hline Female & 43 & 3.79 & 2.756 & & & \\
\hline Male & 170 & 4.25 & 2.796 & & & \\
\hline Total & 213 & 4.15 & 2.788 & & & \\
\hline Marital status & & & & 5.766 & 2,210 & 0.004 \\
\hline Single & 89 & 3.56 & 2.80 & & & \\
\hline Married & 91 & 4.27 & 2.74 & & & \\
\hline Separated/Divorced/Widowed & 33 & 5.42 & 2.48 & & & \\
\hline Total & 213 & 4.15 & 2.79 & & & \\
\hline Education & & & & 0.059 & 1,211 & 0.809 \\
\hline None - Primary & 140 & 4.12 & 2.835 & & & \\
\hline Post-primary & 73 & 4.22 & 2.714 & & & \\
\hline Total & 213 & 4.15 & 2.788 & & & \\
\hline Religion & & & & 3.867 & 2,201 & 0.022 \\
\hline Christians & 189 & 3.97 & 2.79 & & & \\
\hline Islam & 17 & 5.59 & 2.50 & & & \\
\hline Others & 7 & 5.71 & 2.21 & & & \\
\hline Total & 213 & 4.15 & 2.79 & & & \\
\hline Employment status & & & & 6.016 & 2,208 & 0.003 \\
\hline Casual laborer & 31 & 4.84 & 2.81 & & & \\
\hline Employed & 17 & 6.00 & 1.77 & & & \\
\hline Self-employed & 163 & 3.83 & 2.78 & & & \\
\hline Total & 211 & 4.16 & 2.79 & & & \\
\hline Monthly earning & & & & 3.505 & 2,207 & 0.032 \\
\hline Less than 50 & 130 & 4.21 & 2.79 & & & \\
\hline $50-100$ & 51 & 3.43 & 2.83 & & & \\
\hline Over 100 & 29 & 5.10 & 2.41 & & & \\
\hline Total & 210 & 4.14 & 2.79 & & & \\
\hline Family description & & & & 2.231 & 1,208 & 0.137 \\
\hline Abusive/broken home & 34 & 4.79 & 2.496 & & & \\
\hline Happy home & 176 & 4.02 & 2.827 & & & \\
\hline Total & 210 & 4.14 & 2.786 & & & \\
\hline
\end{tabular}

\section{Reported type of alcoholic drink consumed by the} respondents

The majority of the respondents reported consuming chang'aa regardless of gender (Table 4). However, further analyses showed respondents earning a monthly income of over 50 USD were more likely ( $p<0.05$ ) to consume beer and wine. Specifically, those earning monthly income of $50-100$ or above 100 USD were 7.1 and 4.8 times likely to consume beer, respectively. Monthly earners of $50-100$ USD or above 100 USD were 6.3 and 6.2 times more likely to consume wine. Additionally, those earning over 50-100 USD were likely to consume muratina and busaa, however, those with high income were less likely to consume Changaa. 
Table 4. Relationship between income and the type of alcohol consumed among respondents

\begin{tabular}{|c|c|c|c|c|}
\hline \multirow{2}{*}{$\begin{array}{l}\text { Monthly } \\
\text { earning (in } \\
\text { USD) }\end{array}$} & \multicolumn{2}{|c|}{ Alcohol type } & \multirow{2}{*}{ Total } & \multirow{2}{*}{$\operatorname{AOR}(95 \% \mathrm{CI})$} \\
\hline & Yes & No & & \\
\hline \multicolumn{5}{|c|}{ Chang'aa } \\
\hline Less than 50 & $\begin{array}{r}122 \\
(93.8)\end{array}$ & $8(6.2)$ & $130(100)$ & Reference \\
\hline $50-100$ & $49(96.1)$ & $2(3.9)$ & $51(100)$ & $0.2(0.07-0.63)^{*}$ \\
\hline Over 100 & $22(75.9)$ & $7(24.1)$ & $29(100)$ & $0.1(0.03-0.67)^{*}$ \\
\hline \multicolumn{5}{|c|}{ Muratina } \\
\hline Less than 50 & $16(12.3)$ & 114 (87.7) & $130(100)$ & Reference \\
\hline $50-100$ & $10(19.6)$ & $41(80.4)$ & $51(100)$ & $2.7(1.03-7.15)^{*}$ \\
\hline Over 100 & $8(27.6)$ & $21(72.4)$ & $29(100)$ & $1.6(0.54-4.55)$ \\
\hline \multicolumn{5}{|c|}{ Busaa } \\
\hline Less than 50 & $11(8.5)$ & $119(91.5)$ & $130(100)$ & Reference \\
\hline $50-100$ & $4(7.8)$ & $47(92.2)$ & $51(100)$ & $4.9(1.79-13.24)^{*}$ \\
\hline Over 100 & $9(31)$ & $20(69)$ & $29(100)$ & $5.3(1.46-19.19)^{*}$ \\
\hline \multicolumn{5}{|c|}{ Beer } \\
\hline Less than 50 & $9(6.9)$ & $121(93.1)$ & $130(100)$ & Reference \\
\hline $50-100$ & $5(9.8)$ & $46(90.2)$ & $51(100)$ & $7.1(2.55-19.67)^{*}$ \\
\hline Over 100 & $10(34.5)$ & $19(65.5)$ & $29(100)$ & $4.8(1.46-16.06)^{*}$ \\
\hline \multicolumn{5}{|c|}{ Wine } \\
\hline Less than 50 & $10(7.7)$ & $120(92.3)$ & $130(100)$ & Reference \\
\hline $50-100$ & $4(7.8)$ & 47 (92.2) & $51(100)$ & $6.3(2.32-17.19)^{*}$ \\
\hline Over 100 & $10(34.5)$ & $19(65.5)$ & $29(100)$ & $6.2(1.73-22.16)^{*}$ \\
\hline \multicolumn{5}{|c|}{ Spirits } \\
\hline Less than 50 & $14(10.8)$ & $116(89.2)$ & $130(100)$ & Reference \\
\hline $50-100$ & $5(9.8)$ & $46(90.2)$ & $51(100)$ & $3.2(1.18-8.45)^{*}$ \\
\hline Over 100 & $8(27.6)$ & $21(72.4)$ & $29(100)$ & $3.5(1.02-12.00)^{*}$ \\
\hline
\end{tabular}

Presence of family members who consumed alcohol Most respondents reported having family members who consumed alcohol (Table 5). Fathers and siblings were mostly reported to have been consumers of alcohol. Further analyses showed, respondents whose father con- sumed alcohol were 5.5 times likely to drink more than three drinks per day for more days per week $(t=2.284$, $\mathrm{df}=140, \mathrm{p}=0.024)$. Similarly, those with drinking siblings were more likely to consume more than 3 drinks per day as well as engage in drinking for more days in a week $(\mathrm{t}=$ 5.86, $\mathrm{df}=140, \mathrm{p}<0.001)$. 
Table 5: Relationship between presence of a drinking family member and alcohol use

\begin{tabular}{|c|c|c|c|c|c|c|c|c|}
\hline \multirow{2}{*}{$\begin{array}{l}\text { Family } \\
\text { member } \\
\text { drinks } \\
\text { alcohol }\end{array}$} & \multicolumn{2}{|c|}{ Drinks per day } & \multirow{2}{*}{$\operatorname{AOR}(95 \% \mathrm{CI})$} & \multicolumn{2}{|c|}{$\begin{array}{c}\text { Drinking } \\
\text { days per } \\
\text { week }\end{array}$} & \multirow{2}{*}{$\mathrm{t}$} & \multirow{2}{*}{ df } & \multirow{2}{*}{$\mathrm{P}$} \\
\hline & $\begin{array}{l}\text { More } \\
\text { than } 3 \\
\text { drinks }\end{array}$ & $\begin{array}{c}1-3 \\
\text { drinks }\end{array}$ & & Mean & SD & & & \\
\hline Father & & & & & & 2.284 & 140 & 0.024 \\
\hline Yes & $\begin{array}{r}10 \\
(10.3)\end{array}$ & $87(89.7)$ & Reference & 3.29 & 2.679 & & & \\
\hline No & $\begin{array}{r}17 \\
(38.6)\end{array}$ & $27(61.4)$ & $5.5(2.2-13.4)^{*}$ & 4.41 & 2.781 & & & \\
\hline Mother & & & & & & 0.702 & 140 & 0.484 \\
\hline Yes & $1(7.1)$ & $13(92.9)$ & Reference & 3.14 & 2.507 & & & \\
\hline No & $\begin{array}{r}26 \\
(20.5)\end{array}$ & $101(79.5)$ & $3.3(0.4-26.8)$ & 3.69 & 2.78 & & & \\
\hline Siblings & & & & & & 5.859 & 140 & 0.000 \\
\hline Yes & $\begin{array}{r}20 \\
(64.5)\end{array}$ & $11(35.5)$ & Reference & 5.94 & 2.081 & & & \\
\hline No & $7(6.4)$ & 103 (93.6) & $\begin{array}{c}0.04(0.01- \\
0.11)^{*}\end{array}$ & 2.99 & 2.571 & & & \\
\hline Wife & & & & & & 1.132 & 140 & 0.260 \\
\hline Yes & $2(40.0)$ & $3(60.0)$ & Reference & 5 & 2.739 & & & \\
\hline No & $\begin{array}{r}25 \\
(18.4)\end{array}$ & $111(81.6)$ & $0.3(0.05-2.13)$ & 3.58 & 2.748 & & & \\
\hline Children & & & & & & 1.299 & 140 & 0.196 \\
\hline Yes & $3(60.0)$ & $2(40.0)$ & Reference & 5.2 & 2.49 & & & \\
\hline No & $\begin{array}{r}24 \\
(17.6)\end{array}$ & $112(82.4)$ & $\begin{array}{c}0.14(0.02- \\
0.90)^{*}\end{array}$ & 3.58 & 2.751 & & & \\
\hline Total & $\begin{array}{r}27 \\
(19.1)\end{array}$ & $114(80.9)$ & & 3.63 & 2.751 & & & \\
\hline
\end{tabular}

AOR - Adjusted Odds Ratio; CI - Confidence Interval; * - p-value $<0.05$

Reported reasons for alcohol consumption among dents with post-primary education and those from happy the respondents homes were 0.5 and 0.4 less likely to have stress, respecRespondents advanced several reasons for engaging in tively. However, those earning a monthly income of $50-$ alcohol consumption including; stress, peer pressure, 100 USD were 4.1 times more likely to have stress (Table fun, and addiction. Further analysis revealed that respon- 6). 
Table 6: Reported reasons for alcohol use among respondents

\begin{tabular}{|c|c|c|c|c|}
\hline \multirow{2}{*}{ Characteristic } & \multicolumn{2}{|c|}{ Stress } & \multirow{2}{*}{ Total } & \multirow{2}{*}{$\mathrm{AOR}(95 \% \mathrm{CI})$} \\
\hline & Yes & No & & \\
\hline \multicolumn{5}{|l|}{ Marital status } \\
\hline Single & $54(59.3)$ & $37(40.7)$ & $91(100)$ & Reference \\
\hline Married & $36(39.6)$ & $55(60.4)$ & $91(100)$ & $0.5(0.2-1.2)$ \\
\hline Separated/Divorced/Widowed & $14(43.8)$ & $18(56.3)$ & $32(100)$ & $1.2(0.5-2.7)$ \\
\hline \multicolumn{5}{|l|}{ Education } \\
\hline None - Primary & $77(54.6)$ & $64(45.4)$ & 141(100) & Reference \\
\hline Post-primary & $27(37)$ & $46(63)$ & $73(100)$ & $0.5(0.3-0.9)^{*}$ \\
\hline \multicolumn{5}{|l|}{ Monthly earning (USD) } \\
\hline Over 100 & $7(23.3)$ & $23(76.7)$ & $30(100)$ & Reference \\
\hline $50-100$ & $23(45.1)$ & 28(54.9) & $51(100)$ & $4.1(1.7-10.3)^{*}$ \\
\hline Less than 50 & $73(55.7)$ & $58(44.3)$ & $131(100)$ & $1.5(0.8-2.9)$ \\
\hline \multicolumn{5}{|l|}{ Family description } \\
\hline Abusive/broken home & $22(64.7)$ & $12(35.3)$ & $34(100)$ & Reference \\
\hline Happy home & $80(44.9)$ & $98(55.1)$ & $178(100)$ & $0.4(0.2-0.96)^{*}$ \\
\hline Total & $104(48.6)$ & $110(51.4)$ & $214(100)$ & \\
\hline
\end{tabular}

Introduction into alcohol consumption among the Further analysis revealed that the young $\left(\chi^{2}=18.55, \mathrm{p}\right.$ respondents

A majority of the respondents reported having been in- casual laborer $\left(\chi^{2}=15.28, p=0.008\right)$ were likely to have troduced by their friends into drinking alcohol (Table 7). been introduced into drinking by friends.

Table 7: Introduction into alcohol consumption among the respondents

\begin{tabular}{|c|c|c|c|c|c|c|}
\hline \multirow[b]{2}{*}{ Characteristic } & \multicolumn{4}{|c|}{ Introduced to take alcohol by } & \multirow[b]{2}{*}{ Total } & \multirow{2}{*}{$\begin{array}{c}\text { Fisher's } \\
\text { Exact } \\
\text { Test }\end{array}$} \\
\hline & Friends & $\begin{array}{c}\text { Own } \\
\text { initiative }\end{array}$ & Siblings & Relatives & & \\
\hline Age & & & & & & 18.55 \\
\hline Less than 30 years & $99(90.0)$ & $2(1.8)$ & $2(1.8)$ & $7(6.4)$ & $110(100)$ & \\
\hline $31-40$ years & $62(82.7)$ & $6(8.0)$ & $1(1.3)$ & $6(8.0)$ & $75(100)$ & \\
\hline Over 40 years & $16(59.3)$ & $2(7.4)$ & $4(14.8)$ & $5(18.5)$ & $27(100)$ & \\
\hline Marital status & & & & & & 16.56 \\
\hline Single & $81(92.0)$ & $2(2.3)$ & $1(1.1)$ & $4(4.5)$ & $88(100)$ & \\
\hline Married & $76(83.5)$ & $4(4.4)$ & $4(4.4)$ & $7(7.7)$ & $91(100)$ & \\
\hline Separated/Divorced/Widowed & $20(60.6)$ & $4(12.1)$ & $2(6.1)$ & $7(21.2)$ & $33(100)$ & \\
\hline Employment status & & & & & & 15.28 \\
\hline Casual laborer & $27(87.1)$ & $2(6.5)$ & $1(3.2)$ & $1(3.2)$ & $31(100)$ & \\
\hline Employed & $9(52.9)$ & $2(11.8)$ & $3(17.6)$ & $3(17.6)$ & $17(100)$ & \\
\hline Self-employed & $140(85.9)$ & $6(3.7)$ & $3(1.8)$ & $14(8.6)$ & $163(100)$ & \\
\hline Total & $177(83.5)$ & $10(4.7)$ & $7(3.3)$ & $18(8.5)$ & $212(100)$ & \\
\hline
\end{tabular}


Knowledge on the negative effects of alcohol among the respondents

Most respondents acknowledged alcohol was harmful.

Those with post-primary education were 0.5 time less likely to acknowledge the negative effects of alcohol (Table 8). The self-employed and those earning a monthly income of $50-100$ USD were 5.9 and 2.5 times more likely to acknowledge the negative effects of alcohol, respectively.

Table 8: Knowledge on the negative effects of alcohol among the respondents

\begin{tabular}{|c|c|c|c|c|}
\hline \multirow{2}{*}{ Variables } & \multicolumn{2}{|c|}{ Know alcohol is not good } & \multirow{2}{*}{ Total } & \multirow{2}{*}{$\operatorname{AOR}(95 \% \mathrm{CI})$} \\
\hline & Yes & No & & \\
\hline \multicolumn{5}{|l|}{ Education } \\
\hline $\begin{array}{l}\text { None - } \\
\text { Primary }\end{array}$ & $123(87.9)$ & $17(12.1)$ & $140(100)$ & Reference \\
\hline Post-primary & $55(77.5)$ & $16(22.5)$ & $71(100)$ & $0.5(0.2-1.0)^{*}$ \\
\hline Total & $178(84.4)$ & $33(15.6)$ & $211(100)$ & \\
\hline \multicolumn{5}{|l|}{$\begin{array}{l}\text { Employment } \\
\text { status }\end{array}$} \\
\hline Casual laborer & $23(76.7)$ & $7(23.3)$ & $30(100)$ & Reference \\
\hline Employed & $9(56.3)$ & $7(43.8)$ & $16(100)$ & $2.3(0.9-6.1)$ \\
\hline Self-employed & $144(88.3)$ & $19(11.7)$ & $163(100)$ & $5.9(2.0-17.7)^{*}$ \\
\hline Total & $176(84.2)$ & $33(15.8)$ & $209(100)$ & \\
\hline \multicolumn{5}{|l|}{$\begin{array}{l}\text { Monthly } \\
\text { earning (USD) }\end{array}$} \\
\hline Over 100 & $20(69.0)$ & $9(31.0)$ & $29(100)$ & Reference \\
\hline $50-100$ & $47(94.0)$ & $3(6.0)$ & $50(100)$ & $2.5(1.0-6.2)^{*}$ \\
\hline Less than 50 & $109(84.5)$ & $20(15.5)$ & $129(100)$ & $0.3(0.1-1.2)$ \\
\hline Total & $176(84.6)$ & $32(15.4)$ & $208(100)$ & \\
\hline
\end{tabular}

Attempts to stop consuming alcohol by respondents Of the respondents, $58.5 \%$ reported having attempted to stop alcohol consumption. Furthermore, those that were married were 3.3 more likely to have attempted to stop (Table 9). However, other socio-demographic factors did not yield statistical difference on the attempt to stop alcohol consumption.

Table 9: Relationship between attempts to stop alcohol use and social characteristics

\begin{tabular}{lccrc}
\hline \multirow{2}{*}{ Variable } & \multicolumn{2}{c}{ Tried to stop } & \multirow{2}{*}{ Total } & AOR $(95 \% \mathrm{CI})$ \\
\cline { 2 - 3 } & \multicolumn{1}{c}{ Yes } & No & & \\
\hline Marital status & & & 90 & Reference \\
Single & $43(47.8)$ & $47(52.2)$ & $(100)$ & \\
Married & $57(63.3)$ & $33(36.7)$ & 90 & $3.3(1.3-8.1)^{*}$ \\
Separated/Divorced/Widowed & $24(75.0)$ & $8(25.0)$ & 32 & $1.7(0.7-4.3)$ \\
Total & $124(58.5)$ & $88(41.5)$ & 212 & \\
\hline
\end{tabular}

AOR - Adjusted Odds Ratio; CI - Confidence Interval; * - p-value $<0.05$ 
Reasons for attempting to stop alcohol consumption There were various reasons given for attempt to stop alcohol consumption including; addiction (42.3\%), harmfulness $(38.5 \%)$, as well as not being beneficial $(19.2 \%)$.

\section{Source of advice to stop alcohol use}

Most of the respondents reported having been advised to stop consumption of alcohol. The advice was mainly from family members $(68.0 \%)$, professionals $(18.7 \%)$ and friends $(13.3 \%)$.

\section{Respondent feelings after waking up}

The respondents expressed different feelings on wak- ing up associated with alcohol use including; tiredness (45.2\%), need to take alcohol (29.5\%), headache (21.4\%) and okay $(3.8 \%)$, respectively.

\section{Respondents level of responsibility}

Of the respondents, $62 \%$ had children $(3 \pm 2$ ) (Mean \pm $\mathrm{SD})$, of which they were likely to consume more than three drinks in a day and more frequently $(\mathrm{t}=3.93$, $\mathrm{df}=209, \mathrm{p}<0.001)$ (Table 10). Those who did not live with their children were 3.9 times likely to consume more than three drinks per day and drank more frequently $(\mathrm{t}=$ 2.481, $\mathrm{df}=130, \mathrm{p}<0.014)$. Moreover, those who did not take care of their children were 2.4 times likely to drink more per day and more frequently.

Table 10: Relationship between family responsibilities and level of alcohol use among respondents

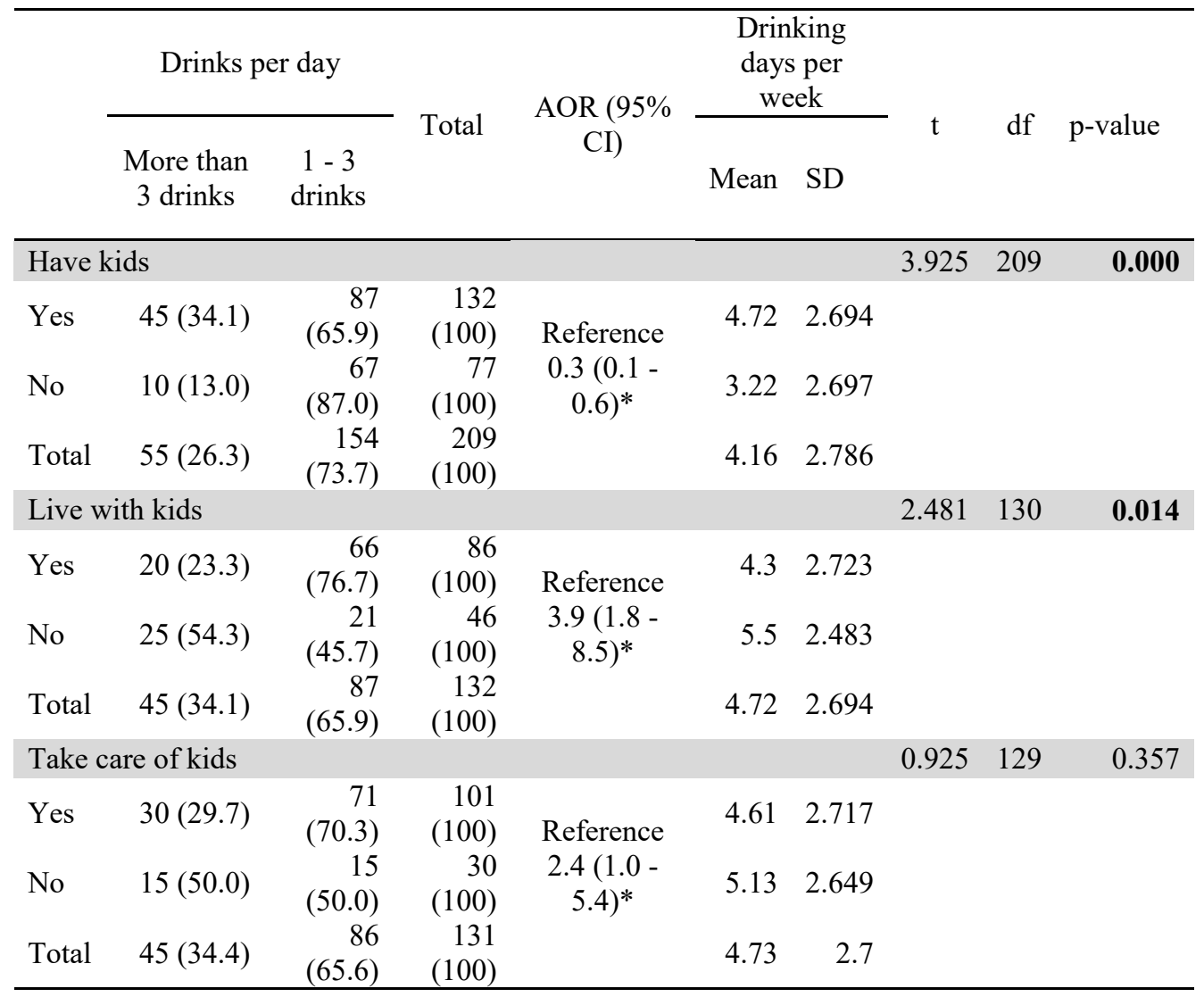

AOR - Adjusted Odds Ratio; CI - Confidence Interval; * - p-value $<0.05$

\section{Availability of support systems for addressing harm- ful use of alcohol}

Respondents reported existence of support systems for harmful alcohol use including; religious institutions, family and friends, and youth centers (Table 11). Further, those with low education attainment $\left(\chi^{2}=18.16, \mathrm{df}=3\right.$, $\mathrm{p}<0.001)$, unmarried (single) $\left(\chi^{2}=32.14, \mathrm{p}<0.001\right)$ and those that consumed less drinks per day were more likely to seek support from religious institutions. Majority of respondents expressed that the support systems were helpful, with religious institutions more likely $\left(\chi^{2}\right.$ $=36.62, \mathrm{p}<0.001)$ to be rated high. 
Table 11: Reported support systems for addressing harmful use of alcohol

\begin{tabular}{|c|c|c|c|c|c|c|c|c|}
\hline \multirow{2}{*}{ Characteristic } & \multicolumn{4}{|c|}{ Support system } & \multirow[t]{2}{*}{ Total } & \multirow[t]{2}{*}{$\chi^{2}$} & \multirow[t]{2}{*}{ df } & \multirow{2}{*}{$\begin{array}{c}\mathrm{p}- \\
\text { value }\end{array}$} \\
\hline & $\begin{array}{l}\text { Family } \\
\text { and } \\
\text { friends }\end{array}$ & $\begin{array}{c}\text { Religious } \\
\text { bodies }\end{array}$ & $\begin{array}{l}\text { Youth } \\
\text { centers }\end{array}$ & None & & & & \\
\hline Гduntion & & & & & & & & $<$ \\
\hline & & & & & & 18.16 & 3 & 0.001 \\
\hline None - Primary & $13(9.4)$ & $95(68.3)$ & $7(5.0)$ & $24(17.3)$ & $139(100)$ & & & \\
\hline Post-primary & $15(21.4)$ & $35(50.0)$ & $13(18.6)$ & $7(10.0)$ & $70(100)$ & & & \\
\hline Total & $28(13.4)$ & $130(62.2)$ & $20(9.6)$ & $31(14.8)$ & $209(100)$ & & & \\
\hline Marital status* & & & & & & 32.14 & & $\begin{array}{l}< \\
0.001\end{array}$ \\
\hline Single & $5(5.6)$ & $68(76.4)$ & $8(9.0)$ & $8(9.0)$ & $89(100)$ & & & \\
\hline Married & $14(15.7)$ & $54(60.7)$ & $10(11.2)$ & $11(12.4)$ & $89(100)$ & & & \\
\hline Separated/Divorced/Widowed & $9(29.0)$ & $8(25.8)$ & $2(6.5)$ & $12(38.7)$ & $31(100)$ & & & \\
\hline Total & $28(13.4)$ & $130(62.2)$ & $20(9.6)$ & $31(14.8)$ & $209(100)$ & & & \\
\hline Drinks per day & & & & & & 61.62 & 3 & $\begin{array}{l}< \\
0.001\end{array}$ \\
\hline 1 - 3 drinks & $14(9.2)$ & $118(77.1)$ & $7(4.6)$ & $14(9.2)$ & $153(100)$ & & & \\
\hline More than 3 drinks & $14(26.4)$ & $9(17.0)$ & $13(24.5)$ & $17(32.1)$ & $53(100)$ & & & \\
\hline Total & $28(13.6)$ & $127(61.7)$ & $20(9.7)$ & $31(15)$ & $206(100)$ & & & \\
\hline Support system help* & & & & & & 38.62 & & $\begin{array}{l}< \\
0.001\end{array}$ \\
\hline Disagree & $5(25.0)$ & $7(35.0)$ & $6(30.0)$ & $2(10.0)$ & $20(100)$ & & & \\
\hline Not sure & $7(46.7)$ & $3(20.0)$ & $3(20.0)$ & $2(13.3)$ & $15(100)$ & & & \\
\hline Agree & $16(10.7)$ & $120(80.0)$ & $11(7.3)$ & $3(2.0)$ & $150(100)$ & & & \\
\hline Total & $28(15.1)$ & $130(70.3)$ & $20(10.8)$ & $7(3.8 .0)$ & $185(100)$ & & & \\
\hline
\end{tabular}

* Fisher's Exact Test

\section{Discussion}

Our findings revealed that: individuals who were older, married, separated/divorced/widowed, of high educational level and earnings consumed more alcohol per session and more frequently; low income earners consumed unrecorded drinks while high earners drank recorded alcohol (beer and wines); families with a drinking father and drinking siblings were likely to consume more; individuals who reported consuming alcohol but were single, attained low educational, low earners and from broken families attributed their drinking to stress; the younger, unmarried, and casual laborers were likely to have been introduced to drinking by friends; alcohol-related negative effects were reported by individuals with low educational attainment, ernings and the self-employed; the separated, divorced and widowed were likely to have attempted to stop alcohol use; individuals with family responsibilities were likely to drink less; and the support offered by religious institutions were perceived to be useful among individuals with low educational attainment, unmarried and those who consumed less alcohol. These findings are elaborated in the subsequent narrative.

The older (above 31 years) individuals consumed more drinks per session as well as more frequently. Adults above 31 years old have the financial capability and can afford 
frequent and high amount of alcohol, in trying to quench their bodies which may have been exposed to long-term alcohol intake. Evidence link increased consumption of alcohol with increasing age ${ }^{49,50}$, with other reports showing later teenage and early adult years being associated with heaviest drinking ${ }^{51,52}$. The findings concur with regional reports from Uganda ${ }^{8}$, Ethiopia ${ }^{53}$, Ghana ${ }^{54}$ and Nigeria ${ }^{55}$ where the older drank heavily per drinking session than the young. Similarly, WHO global reports show older drinkers consume alcohol more frequently than other age groups ${ }^{4}$. The observed drinking pattern among the older individuals is despite their vulnerability to alcohol related complications associated with diminished volume of distribution as a result of decreased lean body mass ${ }^{56}$, as well as increased sensitivity to blood alcohol level ${ }^{57}$.

Interesting, married individuals consumed more alcoholic per session and frequently regardless of gender. Married individuals are more likely to be financially secured and socially involved which might contribute to their drinking behavior as compared to people who are single. However, previous studies show marriage is associated with less drinking in amount and frequency in both men and women linked to direct spousal regulation ${ }^{58-60}$, as well as indirect instrumental, emotional, and informational support that pacifies psychological distress ${ }^{61}$. Additionally, marriage regulate stress and offer greater life satisfaction ${ }^{62}$ and social control ${ }^{63}$. On the contrary, the separated, divorced or widowed loose social support and social control, as well as have increased stress during dissolution that may contribute to heavy alcohol use ${ }^{63-65}$. Men are more vulnerable to this risk and often engage in externalizing behaviors such as heavy drinking to cope with stress ${ }^{66,67}$. Indeed, in comparison with married adults, greater alcohol consumption is characteristic of the divorced 68,69 and the never married ${ }^{70,71}$.

Surprisingly, individuals with higher educational attainment and earnings consume more alcoholic drinks per session as well as drink most days of the week. Educational status has been touted as a marker of social economic status ${ }^{72}$. Those with higher education are formally employed, with higher earnings, thus can afford recorded alcohol. Educational institutions are the main socialization agents, people who spend substantial time in school may commence alcohol consumption as part of socialization, peer pressure, school-related stresses and finan- cial security compared to people who spend less time in schooling ${ }^{73}$. The prolonged schooling acquired drinking behavior could continue into adulthood resulting into alcohol dependence and abuse later in life ${ }^{74,75}$. These findings corroborate regional reports from Uganda, Ethiopia, Ghana and Nigeria that showed individuals with high education drank more ${ }^{54,76-78}$. Similarly, higher socioeconomic status is associated with higher alcohol consumption in older people, with income showing an association between moderate and heavy drinking ${ }^{79-83}$. However, a negative relationship between educational status and/or socioeconomic status and unrecorded alcohol consumption have been reported mainly because of affordability and availability. For example, in this study individuals with low education attainment and socioeconomic level were reported to drink more unrecorded alcohol. The findings are supported by reports from Uganda that have shown that youth participating in vocational training programs to build their skills and knowledge were less likely to report drinking of alcohol than those who did not attend such ${ }^{84}$. Related to the aforesaid, socioeconomic deprivation is a significant predictor of unsafe alcohol consumption ${ }^{85}$ with the attendant health consequences. No wonder, consumers of unrecorded alcohol were likely to report alcohol-related negative effects because of the toxicity effects and the tendency to consume high amounts. Similar findings have been adduced from Nigeria where abusers of local brew were found to have lower educational attainment ${ }^{86,87}$. Traditional alcoholic beverages are widely available in rural communities, often at a cost most people can afford for which alcohol pricing has long been recognized as a tool for the control of alcohol abuse ${ }^{88}$.

Adults from families with a drinking father and none drinking siblings were more likely to drink less, while those with both drinking consumed more alcohol. This is supported by the fact that siblings are likely to emulate, support and approve each other drinking behavior as well as copying their fathers. The role of parents in influencing drinking has gained traction for example, very recently in Uganda reports indicate that youth drinking is linked to their parental drinking ${ }^{89}$, underscoring the role of parent in influencing alcohol use behavior. Indeed, paternal drinking problem has been linked with alcohol use and/or abuse in younger adolescents as well ${ }^{90-93}$. The risk of adolescent alcohol misuse is positively associated with increased alcohol use by parents including parental provision of alcohol, favorable parental attitudes towards 
alcohol use and parental drinking ${ }^{41,94-98}$. Additionally, drinking by siblings ${ }^{99-103}$, even when unrelated biologically 104, is associated with alcohol use and/or abuse among adolescents and young adults.

Individuals who are young, unmarried, and casual laborers were likely to have been introduced to drinking by friends. Social groupings and support from friends on matters lifestyle are very important and considered as group social and moral norms. This is consistent with evidence that the young people tend to form an identity independent from their families and foster tighter bonds with their friends during adolescence. Indeed, the friends' drinking patterns are considered to be the strongest predictors of adolescents' and young adults' alcohol use mainly because of peer influence ${ }^{37,41,100,103,105-1118}$. The influence is also determined by the kind of bond, for example the stronger the social interaction the more the likelihood of taking alcohol frequently ${ }^{111,112}$.

The adults who reported consuming alcohol but were single, of low educational attainment and earnings as well as those from broken families attributed their drinking to stress. Alcohol use has been used dysfunctionally to wade off stress and distress among individuals. This is supported by evidence that high prevalence of alcohol use is associated with psychological distress (anxiety-induced sleeplessness and/or depression) among adolescent students in Asia ${ }^{113}$, poor life satisfaction ${ }^{114,115}$ as well as psychological stress related to heavy drinking ${ }^{55,62,116}$. Men have been attributed with increased alcohol consumption to overcome societal stresses ${ }^{117}$. Elsewhere, frustrations associated with work topped the list of reasons adduced for drinking ${ }^{118}$. Thus it is from aforesaid stress and related problems that motivate the alcohol consumers seek support from religious institutions. Indeed, the support was perceived to be useful among those who sought religious intervention.

Our study holds a number of limitations. The assessment of alcohol consumption was retrospective thus recall bias may not have been completely eliminated. However, such bias may not have been substantial as we collected data on alcohol consumption in the last 30 days. In addition, the study did not assess the quantity of alcohol intake an important measure for both recorded as well as unrecorded alcohol consumption. Finally, this was a cross-sectional study and because of the design, the causal relationship cannot be strongly established.

\section{Conclusion}

Social economic status is a predictor of the category of alcoholic drink and drinking patterns. Both parental and sibling alcohol consumption is a strong determinant for other sibling drinking. Stress is a contributory factor to consumption of alcohol among adults of low socioeconomic status, single and those from dysfunctional families as well as suffer alcohol-related negative effects. Friends play key role in introducing their peers to alcohol use. However, marriage and family responsibility appears to be protective against high alcohol consumption. Additionally, religious institutional support is perceived to be useful among individuals with low educational level, unmarried and those who consumed less alcohol.

Our findings show that socio-demographic, economic, being married or separated, familial, social interactions and stress are associated with harmful alcohol use among adults in slum settings in Kenya. However, being a responsible family person is a protective factor against abuse of alcohol. Interventional programs involving young adolescents, families, communities, poverty alleviation, social support, and awareness creation can help address the harmful us of alcohol among slum and informal dwellers in Kenya.

\section{Declarations}

\section{Ethics approval and consent to participate}

Informed consent was obtained from the respondents before they participated in the study. Ethical approval to conduct the study was obtained from Kenyatta National Hospital-University of Nairobi Ethical Review Committee (KNH-UoN ERC) (Approval number UP365/05/2016) and permission sought from the Githurai Assistant County Commissioner (Ref. KASD/ ADM/1/1VOL.5/192).

\section{Consent for publication}

Not Applicable

\section{Availability of data and material}

The datasets used and analyzed during the current study are available from the corresponding author on reasonable request

\section{Competing interest}

The authors declare that they have no competing interests. 


\section{Funding}

There was no funding for this work

\section{Authors' contributions}

MG and SK conceptualized and designed the study, and drafted the initial manuscript. SM carried out the initial analyses, and reviewed and revised the manuscript. OTO critically reviewed the manuscript and contributed equally to this paper.

\section{Acknowledgements}

The authors acknowledge the support received from the study respondents and the local administration

\section{References}

1. Jacob N, MacArthur GJ, Hickman M, Campbell R. A qualitative investigation of the role of the family in structuring young people's alcohol use. The European Journal of Public Health 2015; 26(1):102-10.

2. WHO, Global strategy to reduce the harmful use of alcohol, Geneva: World Health Organization, 2010. 44 p. 3. Gichangi P, Thenya S, Kamau J, et al., Domestic Violence in Kenya: Report of a Baseline Survey Among Women in Nairobi, Nairobi: Fida Kenya, 2002. 36 p.

4. WHO, Global status report on alcohol and health, 2014, Geneva: World Health Organization, 2014. 392 p.

5. Griswold MG, Fullman N, Hawley C, et al. Alcohol use and burden for 195 countries and territories, 1990-2016: a systematic analysis for the Global Burden of Disease Study 2016. The Lancet 2018; 392(10152):1015-35.

6 . WHO, Global health risks: mortality and burden of disease attributable to selected major risks., Geneva, Switzerland: Geneva, Switzerland, 2009.

7. Pompili M, Serafini G, Innamorati M, et al. Suicidal behavior and alcohol abuse. Int J Environ Res Public Health 2010; 7(4):1392-431.

8. Kabwama SN, Ndyanabangi S, Mutungi G, et al. Alcohol use among adults in Uganda: findings from the countrywide non-communicable diseases risk factor cross-sectional survey. Global Health Action 2016; 9(1):31302.

9. Takahashi R, Wilunda C, Magutah K, et al. Correlates of alcohol consumption in rural western Kenya: A cross-sectional study. BMC Psychiatry 2017; 17(1):175.

10. Mkuu RS, Barry AE, Montiel Ishino FA, Amuta AO. Examining characteristics of recorded and unrecorded alcohol consumers in Kenya. BMC Public Health 2018; 18 (1):1058.

11. Ferreira-Borges C, Rehm J, Dias S, Babor T, Parry CD.
The impact of alcohol consumption on African people in 2012: an analysis of burden of disease. Trop Med Int Health 2016; 21(1):52-60.

12. Reuters. Illegal alcohol kills at least 33 people in Kenya, https://www.reuters.com/article/us-kenya-alcohol-casualties/illegal-alcohol-kills-at-least-33-people-inkenya-idUSBREA450GK20140506. (2014, Accessed 28 April 2018)

13. CNN. Illegal local brew kills 14 in Kenya, http:// edition.cnn.com/2010/WORLD/africa/07/27/ kenya. brew.deaths/. (2015, Accessed 28 April 2018)

14. WHO, Global status report on alcohol and health, 2018, Geneva: World Health Organization, 2018. 472 p. 15. Mkuu RS, Barry AE, Swahn MH, Nafukho F. Unrecorded alcohol in East Africa: A case study of Kenya. Int J Drug Policy 2019; 63:12-7.

16. Kendagor A, Gathecha G, Ntakuka MW, et al. Prevalence and determinants of heavy episodic drinking among adults in Kenya: analysis of the STEPwise survey, 2015. BMC Public Health 2018; 18(Suppl 3):1216.

17. Papas RK, Sidle JE, Wamalwa ES, et al. Estimating alcohol content of traditional brew in Western Kenya using culturally relevant methods: the case for cost over volume. AIDS Behav 2010; 14(4):836-44.

18. Musungu JB and Kosgei PK. Production and consumption of non-standardised alcohol in Kenya: with whom does the Buck stop? Global Journal of Arts, Humanities and Social Science 2015; 3(10):8-16.

19. Samson O, Kariuki M and Mwenje M. Co-occurrence of alcohol, tobacco and other drugs among secondary school students in Kiambu and Nairobi counties Kenya. Int J Educ and Res 2013; 1(3).

20. McAllister, Alcohol consumption among adolescents and young adults, Melbourne, Victoria: Distilled Spirits Industries Council of Australia, 2003.

21. Lancet. Calling time on young people's alcohol consumption. The Lancet 2008; 371(9616):871.

22. WHO, WHO Expert Committee on Problems Related to Alcohol Consumption, Geneva: World Health Organization, 2007.

23. Battjes $\mathrm{R}$ and Jones C. Implications of etiological research for preventive interventions and future research. NIDA Res Monogr 1985; 56:269.

24. Morleo M, Cook PA, Elliott G, Phillips-Howard PA. Parental knowledge of alcohol consumption: A cross sectional survey of 11-17 year old schoolchildren and their parents. BMC Public Health 2013; 13(1):412.

25. Velleman RD, Templeton LJ and Copello AG. The 
role of the family in preventing and intervening with substance use and misuse: a comprehensive review of family interventions, with a focus on young people. Drug and $\mathrm{Al}$ cohol Review 2005; 24(2):93-109.

26. Gabel S, Stallings MC, Young SE, et al. Family variables in substance-misusing male adolescents: The importance of maternal disorder. The American Journal of Drug and Alcohol Abuse 1998; 24(1):61-84.

27. Bellis MA, Hughes K, Morleo M, et al. Predictors of risky alcohol consumption in schoolchildren and their implications for preventing alcohol-related harm. Substance Abuse Treatment, Prevention, and Policy 2007; 2(1):15.

28. Trim RS, Leuthe E and Chassin L. Sibling influence on alcohol use in a young adult, high-risk sample. J Stud Alcohol 2006; 67(3):391-8.

29. Van de Rakt M, Nieuwbeerta P and Apel R. Association of criminal convictions between family members: Effects of siblings, fathers and mothers. Crim Behav Ment Health 2009; 19(2):94-108.

30. Webb JA and Baer PE. Influence of family disharmony and parental alcohol use on adolescent social skills, self-efficacy, and alcohol use. Addict Behav 1995; 20(1):127-35.

31. Mattick RP, Clare PJ, Aiken A, et al. Association of parental supply of alcohol with adolescent drinking, alcohol-related harms, and alcohol use disorder symptoms: a prospective cohort study. The Lancet Public Health 2018; 3(2):e64-e71.

32. Gil AG, Wagner EF and Vega WA. Acculturation, familism, and alcohol use among Latino adolescent males: Longitudinal relations. J Community Psychol 2000; 28(4):443-58.

33. Kroll B. Living with an elephant: Growing up with parental substance misuse. Child \& Family Social Work 2004; 9(2):129-40.

34. Babor T, Caetano R, Casswell S, et al. Alcohol: No Ordinary Commodity-a summary of the second edition. Addiction 2010; 105(5):769-79.

35. Teunissen HA, Spijkerman R, Prinstein MJ, et al. Adolescents' Conformity to Their Peers' Pro-Alcohol and Anti-Alcohol Norms: The Power of Popularity. Alcoholism: Clinical and Experimental Research 2012; 36(7):1257-67. 36. Kelly AB, Chan GC, Toumbourou JW, et al. Very young adolescents and alcohol: Evidence of a unique susceptibility to peer alcohol use. Addict Behav 2012; 37(4):414-9. 37. Bot SM, Engels RC, Knibbe RA, Meeus WH. Friend's drinking behaviour and adolescent alcohol consumption: The moderating role of friendship characteristics. Addict Behav 2005; 30(5):929-47.

38. Ali MM and Dwyer DS. Social network effects in alcohol consumption among adolescents. Addict Behav 2010; 35(4):337-42.

39. Moreno MA and Whitehill JM. Influence of social media on alcohol use in adolescents and young adults. Alcohol research: Current Reviews 2014; 36(1):91.

40. Stead M, MacKintosh AM, McDermott L, Eadie D, Evaluation of the Effectiveness of Drug Education in Scottish Schools, Edinburgh, Scottish Executive Education Department, 2007.

41. Wood MD, Read JP, Mitchell RE, Brand NH. Do parents still matter? Parent and peer influences on alcohol involvement among recent high school graduates. Psychol Addict Behav 2004; 18(1):19.

42. Smith L and Foxcroft D, Drinking in the UK: An exploration of trends, Oxford: Oxford Brookes University, 200928 April 2018. 112 p.

43. Gruber E, DiClemente RJ, Anderson MM, Lodico M. Early drinking onset and its association with alcohol use and problem behavior in late adolescence. Prev Med 1996; 25(3):293-300.

44. Hawkins JD, Graham JW, Maguin E, et al. Exploring the effects of age of alcohol use initiation and psychosocial risk factors on subsequent alcohol misuse. J Stud Alcohol 1997; 58(3):280-90.

45. Hingson RW, Heeren T and Winter MR. Age at drinking onset and alcohol dependence: age at onset, duration, and severity. Arch Pediatr Adolesc Med 2006; 160(7):739-46. 46. ACMD, Pathways to Problems: Hazardous use of tobacco, alcohol and other drugs by young people in the $\mathrm{UK}$ and its implications for policy, UK: Advisory Council on the Misuse of Drugs, 2006.

47. Kenya National Bureau of Statistics and ICF Macro, Kenya Demographic and Health Survey 2014, Calverton, Maryland: KNBS and ICF Macro, 2014. 602 p.

48. Mugenda OM and Mugenda AG, Research Methods: Quantitative and Qualitative Approaches, Nairobi: African Centre for Technology Studies (ACTS), 2003.

49. Ahlström S. Alcohol use and problems among older women and men: A review. Nordic studies on Alcohol and Drugs 2008; 25(2):154-61.

50. Casswell S, Huckle T, Wall M, et al. Policy-relevant behaviours predict heavier drinking and mediate the rela- 
tionship with age, gender and education status: Analysis from the International Alcohol Control study. Drug and Alcohol Review 2018.

51. Jernigan DH, Global status report: alcohol and young people, Geneva: World Health Organization, 2001.

52. Kuntsche E, Rehm J and Gmel G. Characteristics of binge drinkers in Europe. Social Science \& Medicine 2004; 59(1):113-27.

53. Teferra S, Medhin G, Selamu M, et al. Hazardous alcohol use and associated factors in a rural Ethiopian district: a cross-sectional community survey. BMC Public Health 2016; 16(1):218.

54. Yawson AE, Welbeck J, Agyenim BJ, et al. Sociodemographic and Socioeconomic Correlates of Alcohol Use among Older Adults in Ghana. Journal of Alcoholism \& Drug Dependence 2015; 3(202).

55. Abayomi O, Onifade PO, Adelufosi AO, Akinhanmi AO. Psychosocial correlates of hazardous alcohol use among undergraduates in southwestern Nigeria. Gen Hosp Psychiatry 2013; 35(3):320-4.

56. Vestal RE, McGuire EA, Tobin JD, et al. Aging and ethanol metabolism. Clin Pharmacol Ther 1977; 21(3):34354.

57. Adams W, Garry P, Rhyne R, Hunt W, Goodwin J. Alcohol intake in the healthy elderly. Changes with age in a cross-sectional and longitudinal study. J Am Geriatr Soc 1990; 38(3):211-6.

58. Reczek $C$ and Umberson D. Gender, health behavior, and intimate relationships: Lesbian, gay, and straight contexts. Social Science \& Medicine 2012; 74(11):1783-90.

59. Liang $W$ and Chikritzhs T. Brief report: marital status and alcohol consumption behaviours. Journal of Substance Use 2012; 17(1):84-90.

60. Prescott CA and Kendler KS. Associations between marital status and alcohol consumption in a longitudinal study of female twins. J Stud Alcohol 2001; 62(5):589-604. 61. Taylor SE, Repetti RL and Seeman T. Health psychology: what is an unhealthy environment and how does it get under the skin? Annu Rev Psychol 1997; 48(1):411-47.

62 . Uecker JE. Marriage and mental health among young adults. J Health Soc Behav 2012; 53(1):67-83.

63. Reczek C, Pudrovska T, Carr D, Thomeer MB, Umberson D. Marital histories and heavy alcohol use among older adults. J Health Soc Behav 2016; 57(1):77-96.

64. Dinescu D, Turkheimer E, Beam CR, et al. Is marriage a buzzkill? A twin study of marital status and alcohol consumption. J Fam Psychol 2016; 30(6):698.
65. Umberson D, Liu H and Reczek C. Stress and health behaviour over the life course. Advances in Life Course Research 2008; 13:19-44.

66. Courtenay WH. Constructions of masculinity and their influence on men's well-being: a theory of gender and health. Social Science \& Medicine 2000; 50(10):1385401.

67. Sacco P, Bucholz KK and Harrington D. Gender differences in stressful life events, social support, perceived stress, and alcohol use among older adults: results from a national survey. Substance Use \& Misuse 2014; 49(4):45665.

68. Heien D and Pompelli G. Stress, ethnic and distribution factors in a dichotomous response model of alcohol abuse. J Stud Alcohol 1987; 48(5):450-5.

69. Linsky AS, Straus MA and Colby Jr JP. Stressful events, stressful conditions and alcohol problems in the United States: a partial test of Bales's theory. J Stud Alcohol 1985; 46(1):72-80.

70. Power C, Rodgers B and Hope S. Heavy alcohol consumption and marital status: disentangling the relationship in a national study of young adults. Addiction 1999; 94(10):1477-87.

71. Simon RW. Revisiting the relationships among gender, marital status, and mental health. American Journal of Sociology 2002; 107(4):1065-96.

72. Colhoun HM, Hemingway $\mathrm{H}$ and Poulter N. Socio-economic status and blood pressure: an overview analysis. J Hum Hypertens 1998; 12(2):91.

73. Melchior M, Chastang J-F, Goldberg P, Fombonne E. High prevalence rates of tobacco, alcohol and drug use in adolescents and young adults in France: results from the GAZEL Youth study. Addict Behav 2008; 33(1):122-33.

74. DeWit DJ, Adlaf EM, Offord DR, Ogborne AC. Age at first alcohol use: a risk factor for the development of alcohol disorders. Am J Psychiatry 2000; 157(5):745-50.

75. Kraus L, Bloomfield K, Augustin R, Reese A. Prevalence of alcohol use and the association between onset of use and alcohol-related problems in a general population sample in Germany. Addiction 2000; 95(9):1389-401.

76. Ouattara ZD, Koura M, Serme AK, et al. Sociodemographic Factors of Alcohol Consumption in a Population of Hospitalized Patients in Ouagadougou (Burkina Faso). Open Journal of Gastroenterology 2017; 7:96-104.

77. Agabio R, Nioi M, Serra C, Valle P, Gessa GL. Alcohol use disorders in primary care patients in Cagliari, Italy. Alcohol Alcohol 2006; 41(3):341-4. 
78. Ronzani T, Amato T, Silveira P, et al. Alcohol Use Pattern Assessment and Its Relation with Social-Demographic Variables among Patients of Primary Health Care (PHC) Poster Session Pb3: Epidemiology, Phenotyping, Comorbidity and Alcoholism. Alcohol Alcohol 2007; 42(suppl_1):i62-i5.

79. Bonevski B, Regan T, Paul C, Baker AL, Bisquera A. Associations between alcohol, smoking, socioeconomic status and comorbidities: evidence from the 45 and Up Study. Drug and Alcohol Review 2014; 33(2):169-76.

80. Touvier M, Druesne-Pecollo N, Kesse-Guyot E, et al. Demographic, socioeconomic, disease history, dietary and lifestyle cancer risk factors associated with alcohol consumption. Int J Cancer 2014; 134(2):445-59.

81. Kumar K, Kumar S and Singh AK. Prevalence and socio-demographic correlates of alcohol consumption: Survey findings from five states in India. Drug Alcohol Depend 2018; 185:381-90.

82. Lee SJ, Sudore RL, Williams BA, et al. Functional Limitations, Socioeconomic Status, and All-Cause Mortality in Moderate Alcohol Drinkers. J Am Geriatr Soc 2009; 57(6):955-62.

83. Parry CD, Plüddemann A, Steyn K, et al. Alcohol use in South Africa: findings from the first Demographic and Health Survey (1998). J Stud Alcohol 2005; 66(1):91-7.

84. Swahn MH, Buchongo P and Kasirye R. Risky behaviors of youth living in the slums of Kampala: a closer examination of youth participating in vocational training programs. Vulnerable Children and Youth Studies 2018; 13(3):276-90.

85. Rao R, Schofield P and Ashworth M. Alcohol use, socioeconomic deprivation and ethnicity in older people. BMJ Open 2015; 5(8):e007525.

86. Brisibe $\mathrm{S}$ and Ordinioha B. Socio-demographic characteristics of alcohol abusers in a rural Ijaw community in Bayelsa State, South-South Nigeria. Ann Afr Med 2011; 10(2).

87. Stanley P and Odejide A. Socio-demographic and forensic characteristics of alcohol abusers in Jos, Nigeria. Nigerian Journal of Medicine: Journal of the National Association of Resident Doctors of Nigeria 2002; 11(3):113-7.

88. WHO, Global Status Report: Alcohol policy, Geneva Department of Mental Health and Substance Abuse, World Health Organization, 2004.

89. Swahn MH, Culbreth R, Tumwesigye NM, et al. Problem Drinking, Alcohol-Related Violence, and Homelessness among Youth Living in the Slums of Kampala, Uganda. International Journal of Environmental Research and Public Health 2018; 15(6).
90. van der Zwaluw CS, Scholte RH, Vermulst AA, et al. Parental problem drinking, parenting, and adolescent alcohol use. J Behav Med 2008; 31(3):189.

91. Haugland SH, Holmen TL, Ravndal E, Bratberg GH. Parental alcohol misuse and hazardous drinking among offspring in a general teenage population: gender-specific findings from the Young-HUNT 3 study. BMC Public Health 2013; 13(1):1140.

92. Lieb R, Merikangas KR, Höfler M, et al. Parental alcohol use disorders and alcohol use and disorders in offspring: a community study. Psychol Med 2002; 32(1):63-78. 93. Sher KJ, Walitzer KS, Wood PK, Brent EE. Characteristics of children of alcoholics: putative risk factors, substance use and abuse, and psychopathology. J Abnorm Psychol 1991; 100(4):427.

94. Yap MB, Cheong TW, Zaravinos-Tsakos F, Lubman DI, Jorm AF. Modifiable parenting factors associated with adolescent alcohol misuse: a systematic review and meta-analysis of longitudinal studies. Addiction 2017; 112(7):1142-62.

95. Rossow I, Keating P, Felix L, McCambridge J. Does parental drinking influence children's drinking? A systematic review of prospective cohort studies. Addiction 2015; 111(2):204-17.

96. Ryan SM, Jorm AF and Lubman DI. Parenting factors associated with reduced adolescent alcohol use: a systematic review of longitudinal studies. Aust N Z J Psychiatry 2010; 44(9):774-83.

97. Rossow I, Felix L, Keating P, McCambridge J. Parental drinking and adverse outcomes in children: A scoping review of cohort studies. Drug and Alcohol Review 2016; 35(4):397-405.

98. Cleveland $\mathrm{HH}$ and Wiebe RP. The moderation of genetic and shared-environmental influences on adolescent drinking by levels of parental drinking. J Stud Alcobol 2003; 64(2):182-94.

99. Needle R, McCubbin H, Wilson M, et al. Interpersonal influences in adolescent drug use - the role of older siblings, parents, and peers. Int J Addict 1986; 21(7):73966.

100. Ary DV, Tildesley E, Hops H, Andrews J. The influence of parent, sibling, and peer modeling and attitudes on adolescent use of alcohol. Int J Addict 1993; 28(9):85380.

101. Duncan TE, Duncan SC and Hops H. The role of parents and older siblings in predicting adolescent substance use: Modeling development via structural equation latent growth methodology. J Fam Psychol 1996; 10(2):158. 102. D'amico EJ and Fromme K. Health risk behaviors 
of adolescent and young adult siblings. Health Psychol 1997; 16(5):426.

103. Windle M. Parental, sibling, and peer influences on adolescent substance use and alcohol problems. Applied Developmental Science 2000; 4(2):98-110.

104. McGue M, Sharma A and Benson P. Parent and sibling influences on adolescent alcohol use and misuse: evidence from a US adoption cohort. J Stud Alcohol 1996; 57(1):8-18.

105. Burk WJ, Van Der Vorst H, Kerr M, Stattin H. Alcohol use and friendship dynamics: Selection and socialization in early-, middle-, and late-adolescent peer networks. Journal of Studies on Alcohol and Drugs 2012; 73(1):89-98. 106. Petraitis J, Flay BR and Miller TQ. Reviewing theories of adolescent substance use: organizing pieces in the puzzle. Psychol Bull 1995; 117(1):67.

107. Webster RA, Hunter M and Keats JA. Peer and parental influences on adolescents' substance use: a path analysis. Int J Addict 1994; 29(5):647-57.

108. Urberg KA, Değirmencioğlu SM and Pilgrim C. Close friend and group influence on adolescent cigarette smoking and alcohol use. Dev Psychol 1997; 33(5):834.

109. Poelen EA, Scholte RH, Willemsen G, Boomsma DI, Engels RC. Drinking by parents, siblings, and friends as predictors of regular alcohol use in adolescents and young adults: a longitudinal twin-family study. Alcohol Alcohol 2007; 42(4):362-9.

110. Kiuru N, Burk WJ, Laursen B, Salmela-Aro K, Nurmi J-E. Pressure to drink but not to smoke: Disentangling selection and socialization in adolescent peer networks and peer groups. J Adolesc 2010; 33(6):801-12.

111. Deutsch AR, Steinley D and Slutske WS. The role of gender and friends' gender on peer socialization of adolescent drinking: A prospective multilevel social network analysis. J Youth Adolesc 2014; 43(9):1421-35.

112. Tumwesigye NM, Kasirye R and Nansubuga E. Is social interaction associated with alcohol consumption in Uganda? Drug Alcohol Depend 2009; 103(1):9-15.

113. Balogun O, Koyanagi A, Stickley A, Gilmour S, Shibuya K. Alcohol consumption and psychological distress in adolescents: a multi-country study. $J$ Adolesc Health 2014; 54(2):228-34.

114. Murphy JG, McDevitt-Murphy ME and Barnett NP. Drink and be merry? Gender, life satisfaction, and alcohol consumption among college students. Psychol Addict Behav 2005; $19(2): 184$.

115. Paul LA, Grubaugh AL, Frueh BC, Ellis C, Egede LE. Associations between binge and heavy drinking and health behaviors in a nationally representative sample. Addict Behav 2011; 36(12):1240-5.

116. Galea S, Nandi A and Vlahov D. The social epidemiology of substance use. Epidemiol Rev 2004; 26(1):36-52. 117. Demmel $\mathrm{R}$ and Hagen J. The structure of positive alcohol expectancies in alcohol-dependent inpatients. $A d$ diction Research \& Theory 2004; 12(2):125-40.

118. Abiona T, Aloba OO and Fatoye FO. Pattern of alcohol consumption among commercial road transport workers in a semi-urban community in south western Nigeria. East African Medical Journal 2006; 83(9). 